\title{
On sums of odd and even terms of the K-Fibonacci numbers
}

\author{
Yashwant K. Panwar ${ }^{1 *}$, G. P. S. Rathore ${ }^{2}$, Richa Chawla ${ }^{3}$ \\ ${ }^{1}$ Department of Mathematics, Mandsaur Institute of Technology, Mandsaur, India \\ ${ }^{2}$ Department of Mathematical Sciences, College of Horticulture, Mandsaur, India \\ ${ }^{3}$ School of Studies in Mathematics, Vikram University, Ujjain, India \\ *Corresponding author E-mail: yashwantpanwar@gmail.com
}

Copyright $\odot 2014$ Panwar et. al. This is an open access article distributed under the Creative Commons Attribution License, which permits unrestricted use, distribution, and reproduction in any medium, provided the original work is properly cited.

\begin{abstract}
In this paper, we define some properties of sums of k-Fibonacci numbers. Also we present the sum of consecutive members of k-Fibonacci numbers and the same thing for even and for odd k-Fibonacci numbers. Mainly, Binet's formula will be used to establish properties of k-Fibonacci numbers.
\end{abstract}

Keywords: K-Fibonacci Numbers, K-Lucas Numbers, Binet's Formula.

\section{Introduction}

The amount of literature bears witness to the ubiquity of the Fibonacci numbers and the Lucas numbers. Not only are these numbers popular in expository literature because of their beautiful properties, but also the fact that they 'occur in nature' adds to their fascination. The Fibonacci sequence is a source of many nice and interesting identities. The term "Fibonacci numbers" is used to describe the series of numbers generated by the pattern

$0,1,1,2,3,5,8,13,21,34,55,89,144 \ldots$

Where each number in the sequence is given by the sum of the previous two terms. It is well known that the Fibonacci numbers and Lucas numbers are closely related. The term "Lucas numbers" is used to describe the series of numbers generated by the pattern

$2,1,3,4,7,11,18,29,47,76,123,199$..

These numbers are of great importance in the study of many subjects such as Algebra, geometry and number theory itself.

The sequence of Fibonacci numbers $F_{n}$ is defined by

$F_{n}=F_{n-1}+F_{n-2}, n \geq 2$ with $F_{0}=0, F_{1}=1$

The sequence of Lucas numbers $L_{n}$ is defined by

$L_{n}=L_{n-1}+L_{n-2}, n \geq 2$ with $L_{0}=2, L_{1}=1$

The second order recurrence sequence has been generalized in two ways mainly, first by preserving the initial conditions and second by preserving the recurrence relation. The k-Fibonacci numbers defined by Falco'n and Plaza, depending only on one integer parameter $\mathrm{k}$ as follows, for any positive real number $\mathrm{k}$, the $\mathrm{k}$-Fibonacci sequence is defined recurrently by

$F_{k, n+1}=k F_{k, n}+F_{k, n-1}, n \geq 1$ with $F_{k, 0}=0, F_{k, 1}=1$

The k-Lucas numbers defined by Falco'n themselves as well as looking at its close relationship with the k-Fibonacci numbers,

$L_{k, n+1}=k L_{k, n}+L_{k, n-1}, n \geq 1$ with $L_{k, 0}=2, L_{k, 1}=k$

In the 19th century, the French mathematician Binet devised two remarkable analytical formulas for the Fibonacci and Lucas numbers. In our case, Binet's formula allows us to express the Fibonacci numbers and Lucas numbers in function of the roots $\mathfrak{R}_{1} \& \mathfrak{R}_{2}$ of the following characteristic equation, associated to the recurrence relation (3) and (4): 


$$
x^{2}=k x+1
$$

The Binet's formula for k-Fibonacci and k-Lucas numbers are given by

$$
\begin{aligned}
& F_{k, n}=\frac{\mathfrak{R}_{1}^{n}-\mathfrak{R}_{2}^{n}}{\mathfrak{R}_{1}-\mathfrak{R}_{2}} \\
& L_{k, n}=\mathfrak{R}_{1}^{n}+\mathfrak{R}_{2}^{n} \\
& \operatorname{In}(6) \&(7), \mathfrak{R}_{1}=\frac{k+\sqrt{k^{2}+4}}{2}, \mathfrak{R}_{2}=\frac{k-\sqrt{k^{2}+4}}{2} .
\end{aligned}
$$

Also, $\mathfrak{R}_{1}+\Re_{2}=k, \Re_{1} \Re_{2}=-1, \Re_{1}-\Re_{2}=\sqrt{k^{2}+4}$;

$\Re_{1}-1=\frac{k-2+\sqrt{k^{2}+4}}{2}, \Re_{2}-1=\frac{k-2-\sqrt{k^{2}+4}}{2} ; \Re_{1}+1=\frac{k+2+\sqrt{k^{2}+4}}{2}, \Re_{2}+1=\frac{k+2-\sqrt{k^{2}+4}}{2}$;

$\mathfrak{R}_{1}^{2}-1=k \Re_{1}, \Re_{2}^{2}-1=k \Re_{2}$ And $\Re_{1}=\frac{-1}{\mathfrak{R}_{2}} ; \mathfrak{R}_{2}=\frac{-1}{\mathfrak{R}_{1}}$.

In [10], new generalized k-Fibonacci sequences have been introduced and studied. Many of the properties of these sequences are proved by simple matrix algebra. This study has been motivated by the arising of two complex valued maps to represent the two antecedents in an specific four-triangle partition. In [12], Falcon and Plaza k- Fibonacci sequence generalizes, between others, both the classical Fibonacci sequence and the Pell sequence. In this paper many properties of these numbers are deduced and related with the so-called Pascal 2-triangle. New generalized k-Fibonacci sequences have been introduced and studied. Several properties of these numbers are deduced and related with the socalled Pascal 2-triangle. In addition, the generating functions for these k-Fibonacci sequences have been given. [9], Falcon present Lucas triangle and its relationship with the k-Lucas numbers, combinatorial formula for k-Lucas numbers, generating function and defined Properties of the diagonals of the Lucas triangle and the rows of the Lucas triangle. In [8], S. Falcon, study the properties of the k-Lucas numbers and will prove these properties will be related with the k-Fibonaci numbers. From a special sequence of squares of k-Fibonacci numbers, the k-Lucas sequences are obtained in a natural form. S. Falcon, examine some of the interesting properties of the k-Lucas numbers themselves as well as looking at its close relationship with the k-Fibonacci numbers. The k-Lucas numbers have lots of properties, similar to those of k-Fibonacci numbers and often occur in various formulae simultaneously with latter. In [17], Yazlik, Yilmaz and Taskara, investigate some proporties additive of $k$-Fibonacci and $k$-Lucas sequences and obtain new identities on sums of powers these sequences and obtain the recurrence relations for powers of k-Fibonacci and k-Lucas sequences. Also they will be given new formulas for the powers of k-Fibonacci and k-Lucas sequences. Clary and Hemenway [7] discovered factored closed-form expressions for all sums of the form $\sum_{k=1}^{n} F_{r k}^{3}$ where $\mathrm{r}$ is an integer. In [4], Jennings presented some remarkable elementary identities for sums of powers of reciprocals of Fibonacci and Lucas numbers. In [6], Melham proved a theorem involving a sum of products of Fibonacci numbers and corresponding theorem for Lucas numbers. In [1], Alex and Hongwei obtained formula which gave powers of Fibonacci numbers by using the generating functions. In this paper, we present the sum of $p+1$ consecutive members of k-Fibonacci numbers and the same thing for even and for odd k-Fibonacci numbers.

\section{Preliminary notes}

In [16], Rajesh and Leversha define some properties of Fibonacci numbers in odd terms. In [19], Zvonko Čerin defines some sums of squares of odd and even terms of Lucas sequence. In [20], Zvonko Čerin improves some results on sums of squares of odd terms of the Fibonacci sequence by Rajesh and Leversha. In [5], H. Belbachir and F. Bencherif recover and extend all result of Zvonko Čerin [18, 2005] and Zvonko Čerin and Gianella [22, 2006]. In [21] Zvonko Čerin and Gianella defines sums of Pell numbers. In this paper we define sum of $p+1$ consecutive members of kFibonacci numbers.

\section{Main results}

In this section, we prove some formulas for sums of a finite number of consecutive terms of the Fibonacci-Like numbers. First we find the formula for the $\sum_{i=O}^{p} F_{k, v+i}$ when $p \geq 0$ and $v \geq 0$ are integers.

Theorem 3.1: For $p \geq 0$ and $v \geq 0$ the following equality holds: 
$\sum_{i=0}^{p} F_{k, v+i}=\frac{1+k}{k}\left\{F_{k, v+p}-F_{k, v-1}\right\}+F_{k, v+p}+\frac{F_{k, v-2}}{k}$

Proof: By Binet's formula, we have

$$
\begin{aligned}
& \sum_{i=0}^{p} F_{k, v+i}=\sum_{i=0}^{p} \frac{\Re_{1}^{v+i}-\mathfrak{R}_{2}^{v+i}}{\mathfrak{R}_{1}-\mathfrak{R}_{2}} \\
& =\frac{1}{\sqrt{k^{2}+4}}\left[\frac{\mathfrak{R}_{1}^{v+p+1}-\mathfrak{R}_{1}^{v}}{\mathfrak{R}_{1}-1}-\frac{\mathfrak{R}_{2}^{v+p+1}-\mathfrak{R}_{2}^{v}}{\mathfrak{R}_{2}-1}\right] \\
& =\frac{1}{\sqrt{k^{2}+4}}\left[\frac{\left(\Re_{2}-1\right)\left(\Re_{1}^{v+p+1}-\Re_{1}^{v}\right)-\left(\Re_{1}-1\right)\left(\Re_{2}^{v+p+1}-\Re_{2}^{v}\right)}{\left(\Re_{1}-1\right)\left(\Re_{2}-1\right)}\right] \\
& =\frac{1}{\sqrt{k^{2}+4}}\left[\frac{\mathfrak{R}_{1}^{\nu+p}\left(\Re_{1} \Re_{2}\right)-\mathfrak{R}_{1}^{v} \mathfrak{R}_{2}-\mathfrak{R}_{1}{ }^{\nu+p+1}+\mathfrak{R}_{1}{ }^{v}-\mathfrak{R}_{2}{ }^{\nu+p}\left(\mathfrak{R}_{1} \mathfrak{R}_{2}\right)+\mathfrak{R}_{1} \mathfrak{R}_{2}{ }^{v}+\mathfrak{R}_{2}{ }^{\nu+p+1}-\mathfrak{R}_{2}{ }^{v}}{\mathfrak{R}_{1} \mathfrak{R}_{2}-\mathfrak{R}_{1}-\mathfrak{R}_{2}+1}\right] \\
& =\frac{1}{\mathfrak{R}_{1}-\mathfrak{R}_{2}}\left[\frac{-\left(\mathfrak{R}_{1}{ }^{\nu+p}-\mathfrak{R}_{2}{ }^{\nu+p}\right)-\left(\mathfrak{R}_{1}{ }^{v} \mathfrak{R}_{2}-\mathfrak{R}_{1} \mathfrak{R}_{2}{ }^{v}\right)-\left(\mathfrak{R}_{1}{ }^{\nu+p+1}-\mathfrak{R}_{2}{ }^{\nu+p+1}\right)+\left(\mathfrak{R}_{1}{ }^{v}-\mathfrak{R}_{2}{ }^{v}\right)}{-\left(\mathfrak{R}_{1}+\mathfrak{R}_{2}\right)}\right] \\
& =\frac{1}{k}\left[\left(\frac{\mathfrak{R}_{1}^{v+p}-\mathfrak{R}_{2}^{v+p}}{\mathfrak{R}_{1}-\mathfrak{R}_{2}}\right)-\left(\frac{\mathfrak{R}_{1}^{v-1}-\mathfrak{R}_{2}^{v-1}}{\mathfrak{R}_{1}-\mathfrak{R}_{2}}\right)+\left(\frac{\mathfrak{R}_{1}^{v+p+1}-\mathfrak{R}_{2}^{v+p+1}}{\mathfrak{R}_{1}-\mathfrak{R}_{2}}\right)-\left(\frac{\mathfrak{R}_{1}^{v}-\mathfrak{R}_{2}{ }^{v}}{\mathfrak{R}_{1}-\mathfrak{R}_{2}}\right)\right] \\
& =\frac{1}{k}\left[F_{k, v+p}-F_{k, v-1}+k F_{k, v+p}+F_{k, v+p-1}-k F_{k, v-1}+F_{k, v-2}\right] \\
& \sum_{i=0}^{p} F_{k, v+i}=\frac{1+k}{k}\left\{F_{k, v+p}-F_{k, v-1}\right\}+F_{k, v+p}+\frac{F_{k, v-2}}{k}
\end{aligned}
$$

This completes the proof.

Theorem 3.2: For $p \geq 0$ and $v \geq 0$ the following equality holds:

$$
\sum_{i=0}^{p} F_{k, 2 v+2 i}=\frac{1}{k}\left(F_{k, 2 v+2 p+1}-F_{k, 2 v-1}\right)
$$

Proof: By Binet's formula, we have

$$
\begin{aligned}
& \sum_{i=0}^{p} F_{k, 2 v+2 i}=\frac{1}{\mathfrak{R}_{1}-\mathfrak{R}_{2}} \sum_{i=0}^{p} \mathfrak{R}_{1}^{2 v+2 i}-\mathfrak{R}_{2}^{2 v+2 i} \\
& =\frac{1}{\mathfrak{R}_{1}-\mathfrak{R}_{2}}\left\{\frac{\mathfrak{R}_{1}^{2 v+2 p+2}-\mathfrak{R}_{1}^{2 v}}{k \mathfrak{R}_{1}}-\frac{\mathfrak{R}_{2}{ }^{2 v+2 p+2}-\mathfrak{R}_{2}{ }^{2 v}}{k \mathfrak{R}_{2}}\right\} \\
& =\frac{1}{k\left(\Re_{1}-\Re_{2}\right)}\left\{\frac{\mathfrak{R}_{1} \Re_{2}\left(\Re_{1}{ }^{2 v+2 p+1}-\mathfrak{R}_{2}^{2 v+2 p+1}\right)-\left(\Re_{2} \mathfrak{R}_{1}^{2 v}-\mathfrak{R}_{1} \mathfrak{R}_{2}{ }^{2 v}\right)}{\mathfrak{R}_{1} \mathfrak{R}_{2}}\right\} \\
& =\frac{1}{k\left(\Re_{1}-\Re_{2}\right) \Re_{1} \Re_{2}}\left\{-\left(\Re_{1}^{2 v+2 p+1}-\mathfrak{R}_{2}^{2 v+2 p+1}\right)+\left(\Re_{1}^{2 v-1}-\mathfrak{R}_{2}{ }^{2 v-1}\right)\right\} \\
& \sum_{i=0}^{p} F_{k, 2 v+2 i}=\frac{1}{k}\left(F_{k, 2 v+2 p+1}-F_{k, 2 v-1}\right)
\end{aligned}
$$

This completes the proof.

Theorem 3.3: For $p \geq 0$ and $v \geq 0$ the following equality holds:

$$
\sum_{i=0}^{p} F_{k, v+i}^{2}=\frac{1}{k\left(k^{2}+4\right)}\left[L_{k, 2 v+2 p+1}-L_{k, 2 v-1}+k\left\{(-1)^{v+p+1}-(-1)^{v}\right\}\right]
$$


Proof: By Binet's formula, we have

$$
\begin{aligned}
& \sum_{i=0}^{p} F_{k, v+i}^{2}=\sum_{i=0}^{p}\left(\frac{\Re_{1}^{v+i}-\Re_{2}^{v+i}}{\Re_{1}-\Re_{2}}\right)^{2} \\
& =\frac{1}{\left(\Re_{1}-\Re_{2}\right)^{2}} \sum_{i=0}^{p}\left\{\Re_{1}^{2 v+2 i}+\Re_{2}^{2 v+2 i}-2\left(\Re_{1} \Re_{2}\right)^{v+i}\right\} \\
& =\frac{1}{\left(\Re_{1}-\mathfrak{R}_{2}\right)^{2}}\left[\mathfrak{R}_{1}^{2 v} \frac{\mathfrak{R}_{1}^{2 p+2}-1}{\mathfrak{R}_{1}^{2}-1}+\mathfrak{R}_{2}^{2 v} \frac{\mathfrak{R}_{2}^{2 p+2}-1}{\mathfrak{R}_{2}^{2}-1}-2\left(\mathfrak{R}_{1} \mathfrak{R}_{2}\right)^{v} \frac{\left(\mathfrak{R}_{1} \mathfrak{R}_{2}\right)^{p+1}-1}{\mathfrak{R}_{1} \mathfrak{R}_{2}-1}\right] \\
& =\frac{1}{k\left(\Re_{1}-\mathfrak{R}_{2}\right)^{2}}\left[\left(-\mathfrak{R}_{2} \mathfrak{R}_{1}{ }^{2 v+2 p+2}+\mathfrak{R}_{2} \mathfrak{R}_{1}{ }^{2 v}\right)+\left(-\mathfrak{R}_{1} \mathfrak{R}_{2}{ }^{2 v+2 p+2}+\mathfrak{R}_{1} \mathfrak{R}_{2}{ }^{2 v}\right)+k\left\{(-1)^{v+p+1}-(-1)^{v}\right\}\right] \\
& =\frac{1}{k}\left[\left\{\frac{\mathfrak{R}_{1}^{2 v+2 p+1}+\mathfrak{R}_{2}^{2 v+2 p+1}}{\left(\mathfrak{R}_{1}-\mathfrak{R}_{2}\right)^{2}}-\frac{\mathfrak{R}_{1}^{2 v-1}+\mathfrak{R}_{2}{ }^{2 v-1}}{\left(\mathfrak{R}_{1}-\mathfrak{R}_{2}\right)^{2}}+\frac{k}{\left(\mathfrak{R}_{1}-\mathfrak{R}_{2}\right)^{2}}(-1)^{v+p+1}-(-1)^{v}\right\}\right] \\
& \sum_{i=0}^{p} F_{k, v+i}^{2}=\frac{1}{k\left(k^{2}+4\right)}\left[L_{k, 2 v+2 p+1}-L_{k, 2 v-1}+k\left\{(-1)^{v+p+1}-(-1)^{v}\right\}\right]
\end{aligned}
$$

This completes the proof.

Theorem 3.4: For $p \geq 0$ and $v \geq 0$ the following equality holds:

$$
\sum_{i=0}^{p}(-1)^{i} F_{k, v+k}^{2}=\frac{1}{k}\left[(-1)^{p}\left\{F_{k, v+p+1}-F_{k, v+p}\right\}+F_{k, v}-F_{k, v-1}\right]
$$

Proof: By Binet's formula, we have

$$
\begin{aligned}
& \sum_{i=0}^{p}(-1)^{i} F_{k, v+k}^{2}=\sum_{i=0}^{p}(-1)^{i}\left(\frac{\mathfrak{R}_{1}^{v+k}-\mathfrak{R}_{2}^{v+k}}{\mathfrak{R}_{1}-\mathfrak{R}_{2}}\right) \\
& =\frac{1}{\mathfrak{R}_{1}-\mathfrak{R}_{2}}\left[\frac{\mathfrak{R}_{1}^{v}-(-1)^{p+1} \mathfrak{R}_{1}^{v+p+1}}{\mathfrak{R}_{1}+1}-\frac{\mathfrak{R}_{2}^{v}-(-1)^{p+1} \mathfrak{R}_{2}^{v+p+1}}{\mathfrak{R}_{2}+1}\right] \\
& =\frac{1}{\mathfrak{R}_{1}-\mathfrak{R}_{2}}\left[\frac{-\mathfrak{R}_{1}{ }^{\nu-1}+(-1)^{p+1} \mathfrak{R}_{1}{ }^{\nu+p}+\mathfrak{R}_{1}{ }^{v}-(-1)^{p+1} \mathfrak{R}_{1}{ }^{v+p+1}+\mathfrak{R}_{2}{ }^{\nu-1}-(-1)^{p+1} \mathfrak{R}_{2}{ }^{\nu+p}-\mathfrak{R}_{2}{ }^{v}+(-1)^{p+1} \mathfrak{R}_{2}{ }^{v+p+1}}{\left(\mathfrak{R}_{1}+1\right)\left(\mathfrak{R}_{2}+1\right)}\right]
\end{aligned}
$$

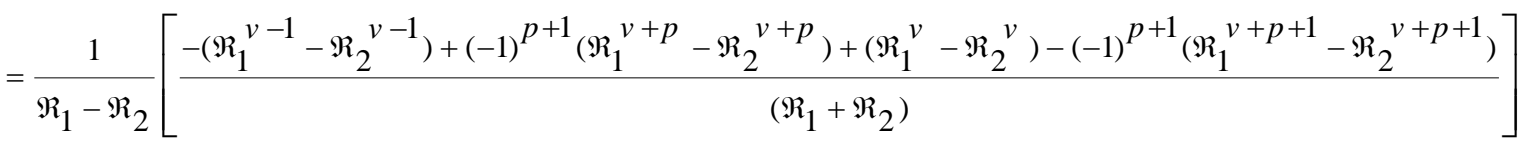

$$
\begin{aligned}
& =\frac{1}{\left(\Re_{1}-\Re_{2}\right)\left(\Re_{1}+\Re_{2}\right)}\left[(-1)^{p}\left(\Re_{1}^{v+p+1}-\Re_{2}^{v+p+1}\right)+\left(\Re_{1}^{v}-\Re_{2}^{v}\right)-\left(\Re_{1}^{v-1}-\Re_{2}^{v-1}\right)-(-1)^{p}\left(\Re_{1}^{v+p}-\Re_{2}^{v+p}\right)\right] \\
& \sum_{i=0}^{p}(-1)^{i} F_{k, v+k}^{2}=\frac{1}{k}\left[(-1)^{p}\left\{F_{k, v+p+1}-F_{k, v+p}\right\}+F_{k, v}-F_{k, v-1}\right]
\end{aligned}
$$

This completes the proof.

\section{Conclusion}

In this paper, we have stated and derived many properties. We define the sum of $p+1$ consecutive members of kFibonacci numbers and the same thing for even and for odd adjacent k-Fibonacci numbers.

\section{References}

[1] A. Chen and H. Chen, "Identities for the Fibonacci Powers", International Journal of Mathemtical Education 39(4) (2008), 534-541.

[2] A. T. Benjamin and J. J. Quinn, "Recounting Fibonacci and Lucas identities", College Math. J., 30(5): (1999), $359-366$.

[3] D. Kalman, R. Mena, The Fibonacci numbers - exposed. Math Mag., 76 (2003), 167-81.

[4] D. Jennings, "On sums the reciprocals of Fibonacci and Lucas Numbers", The Fibonacci Quarterly, 32(1) (1994), 18-21. 
[5] H. Belbachir and F. Bencherif, "Sums of products of generalized Fibonacci and Lucas numbers", arXiv: 0708.2347v1 [math.NT], (2007).

[6] R. S. Melham, "Sums of certain products of Fbonacci and Lucas Numbers", The Fibonacci Quarterly 37(3) (1999), $248-251$.

[7] S. Clarly and D. Hemenway, "On sums of cubes of Fibonacci Numbers", In Applications of Fibonacci Numbers 5 (1993), $123-136$.

[8] S. Falco'n, on the k-Lucas numbers. International Journal of Contemporary Mathematical Sciences, 6(21) (2011), 1039-1050.

[9] S. Falco'n, On the Lucas Triangle and its Relationship with the k-Lucas numbers. Journal of Mathematical and Computational Science, 2(3) (2012), 425-434.

[10] S. Falco'n, Plaza, A.: On the Fibonacci k-numbers. Chaos, Solitons \& Fractals, 32(5) (2007), 1615-1624.

[11] S. Falco'n, Plaza, A.: The k-Fibonacci hyperbolic functions. Chaos, Solitons \& Fractals, 38(2) (2008), 409-20.

[12] S. Falco'n, Plaza, A.: The k-Fibonacci sequence and the Pascal 2-triangle. Chaos, Solitons \&Fractals, 33(1) (2007), 38-49.

[13] S. Vajda, Fibonacci and Lucas numbers, and the golden section. Theory and applications. Chichester: Ellis Horwood limited (1989).

[14] T. Koshy, "Fibonacci and Lucas Numbers with Applications", A Wiley-Interscience Publication, New York, (2001).

[15] V. E. Hoggat, Fibonacci and Lucas numbers. Palo Alto, CA: Houghton, (1969).

[16] V. Rajesh and G. Leversha, "Some properties of odd terms of the Fibonacci sequence", Mathematical Gazette, 88(511): (2004), 85-86.

[17] Y. Yazlik, N. Yilmaz and N. Taskara, "On the Sums of Powers of k-Fibonacci and k-Lucas Sequences" Selçuk J. Appl. Math., Special Issue (2012), 47-50.

[18] Z. Čerin, "Alternating sums of Lucas numbers", Central European Journal of Mathematics, 3(1): (2005), 1-13.

[19] Z. Čerin, "On sums of squares of odd and even terms of the Lucas sequence", Proceedings of the 11th Fibonacci Conference, (to appear).

[20] Z. Čerin, "Properties of odd and even terms of the Fibonacci sequence", Demonstratio Mathematica, 39(1): (2006), 55-60.

[21] Z. Čerin and G. M. Gianella, "On sums of Pell numbers", Acc. Sc. Torino - Atti Sc. Fis. 140, xx-xx. web.math.pmf.unizg.hr/ cerin/c165.pdf, (2006).

[22] Z. Čerin and G. M. Gianella, "On sums of squares of Pell-Lucas numbers", INTEGERS: Electronic Journal of Combinatorial Number Theory, 6, \#A15, (2006). 